\title{
The Effect of the First Computed Tomography Examination on Renal Function During the Initial Cancer Work-up in Newly Diagnosed Cancer Patients without Chronic/Kidney Disease: A Retrospective, Single-institution, Observational Study
}

Sung Han Kim*, Jae Young Joung, Ho Kyung Seo, Kang Hyun Lee and Jinsoo Chung

Department of Urology, Center for Prostate Cancer, National Cancer Center, Goyang, Korea

\begin{abstract}
Purpose: We evaluated the effect of the first computed tomography (CT) examination on renal function during oncologic work-up and the acute kidney injury (AKI) and chronic kidney disease (CKD) rates in newly diagnosed cancer outpatients before cancer therapy.

Materials and methods: From 2005-2013, data from a single health check-up center database were collected on 727 patients without CKD; $592(81.4 \%)$ patients underwent CT. Their renal function was monitored using the serum creatinine $(\mathrm{sCr})$ level and estimated glomerular filtration rate (eGFR) within 3 days before/after the initial CT examination. A maximum increase in the $\mathrm{sCr}$ level $\geq 0.5 \mathrm{mg} / \mathrm{dL}$ from the baseline value following CT was defined as $\mathrm{AKI}$, and an eGFR $<30 \mathrm{ml} / \mathrm{min} / 1.72 \mathrm{~m}^{2}$ as CKD. The predictive factors of AKI and CKD were analyzed.

Results: The median $\mathrm{sCr}(0.96 \mathrm{vs} .1 .06 \mathrm{mg} / \mathrm{dL})$ and eGFR $\left(76.9 \mathrm{vs} .73 .9 \mathrm{~mL} / \mathrm{min} / 1.73 \mathrm{~m}^{2}\right)$ and their median changes from before to after CT ( $\mathrm{sCr} 0.27 \mathrm{mg} / \mathrm{dL}$ and eGFR $\left.-3.15 \mathrm{~mL} / \mathrm{min} / 1.73 \mathrm{~m}^{2}\right)$ were insignificantly different within a median interval time of 1.0 days between the CT and blood examinations $(p>0.05)$. The post-CT rates of AKI and CKD were $13.2 \%(\mathrm{~N}=77)$ and $3.4 \%(\mathrm{~N}=20)$, respectively. In a multivariate analysis, female sex (hazard ratio [HR] 0.757), warfarin therapy (HR 0.371), and pre-CT eGFR (HR 1.010) predicted CKD; male sex (HR 0.288), age (HR 1.010), hypertension (HR 1.464), pre-CT eGFR (HR 1.027), and contrast media use (HR 1.619) predicted AKI $(p<0.05)$.
\end{abstract}

Conclusion: The study identified various risk factors influencing on poor renal function after initial CT for the treatment-naïve cancer patients.

Keywords: Renal function; Computed tomography; Acute kidney injury; Chronic kidney disease; Cancer

\section{Introduction}

With the widespread use of cancer-screening programs and improved scientific imaging, the early detection of cancer enables patients to increase their opportunities of survival, with a longer lifespan. Among diverse and fine imaging techniques such as magnetic resonance and nuclear imaging, computed tomography (CT) is the best choice for diagnosing cancer and determining its stage because of its cost-effectiveness, comfort, objectivity, and ease of performance. However, radiologic exposure and the use of a contrast agent may have nephrotoxic effects on the patient's renal function $[1,2]$.

The nephrotoxic effect of CT has been emphasized in cancer patients, as renal function is an independent factor of survival prognosis. The patient's quality of life after cancer is in remission has also become another important issue in the oncologic field. Clinicians have focused on the renal functional change and protective measures during cancer treatment and follow-up affecting the patients' the best therapeutic outcomes of prognosis. Several measures to preserve renal functional, as well as several predisposing risk factors, have been identified to minimize radiation- and drug-induced nephrotoxicity after multiple CT examinations during cancer therapy and follow-up [3]. Various CT protocols have been established to minimize nephrotoxity in cancer patients, especially those who are predisposed to developing kidney diseases. These protocols include reduced radiation doses and protective medications and fluids that are given before $\mathrm{CT}$ is performed.
To understand what kidney functional changes are produced in cancer patients after multiple CT examinations during cancer therapy, the effects of functional change after the initial CT examination should be evaluated first. This is because cancer patients are exposed to many nephrotoxic examinations and medical therapeutic factors during their cancer therapy and followup. Therefore, this study aimed to investigate the nephrotoxic effect of the first outpatient-based diagnostic CT examination on renal function in newly diagnosed cancer patients who waited for the initiation of cancer therapy during a health check-up screening program. In addition, several predictive risk factors of functional deterioration were statistically assessed to define their relationship with CT-induced chronic kidney disease (CKD) and acute kidney injury $(\mathrm{AKI})$.

*Corresponding author: Sung Han Kim, Department of Urology, Center for Prostate Cancer, National Cancer Center, 323 Ilsan-Ro, Ilsandong-Gu, Goyang-Si, Gyeonggi-Do, 10408, Republic of Korea, Tel: +82-31-920-1706; Fax: +82-31-9202799; E-mail: 12112@ncc.re.kr

Received August 02, 2018; Accepted November 02, 2018; Published November 05, 2018

Citation: Kim SH, Joung JY, Seo HK, Lee KH, Chung J (2018) The Effect of the First Computed Tomography Examination on Renal Function During the Initial Cance Work-up in Newly Diagnosed Cancer Patients without Chronic/Kidney Disease: A Retrospective, Single-institution, Observational Study. J Cancer Sci Ther 10: 332-337. doi: 10.4172/1948-5956.1000564

Copyright: (c) $2018 \mathrm{Kim} \mathrm{SH}$, et al. This is an open-access article distributed unde the terms of the Creative Commons Attribution License, which permits unrestricted use, distribution, and reproduction in any medium, provided the original author and source are credited. 
Citation: Kim SH, Joung JY, Seo HK, Lee KH, Chung J (2018) The Effect of the First Computed Tomography Examination on Renal Function During the Initial Cancer Work-up in Newly Diagnosed Cancer Patients without Chronic/Kidney Disease: A Retrospective, Single-institution, Observational Study. J Cancer Sci Ther 10: 332-337. doi: 10.4172/1948-5956.1000564

\section{Materials and Methods}

\section{Ethics statement}

This retrospective study was approved by the Institutional Review Board of the National Cancer Center (IRB No. NCC2014-0129); the need to obtain informed consent was waived. All of the patients data were anonymized prior to the analysis. All study protocols were performed in accordance with the ethical tenets of the Declaration of Helsinki.

\section{Inclusion and exclusion criteria}

Between 2005 and 2013, data on 1,330 patients who did not have a history of cancer and were aged over 19 years with newly diagnosed, untreated cancer were collected from a single health check-up database at the National Cancer Center between 2005 and 2013. The patients were recruited under the condition that they had completed cancer treatment at the National Cancer Center without being lost to follow-up. I excluded patients who received other imaging work-up examinations (besides CT) before the initial CT examination and at 3-day intervals afterwards $(\mathrm{N}=495)$, those who underwent angiographic $\mathrm{CT}(\mathrm{N}=134)$, and those with a baseline serum creatinine (sCr) level below $1.5 \mathrm{mg} /$ $\mathrm{dL}(\mathrm{N}=112)$ or estimated glomerular filtration rate (eGFR) $<60 \mathrm{~mL} /$ $\mathrm{min} / \mathrm{mm}^{2}(\mathrm{~N}=148)$. Thus, 583 patients were finally enrolled in this study.

\section{Assessment of renal function and the laboratory definition of AKI after CT}

Each patient's renal function was evaluated using the sCr level, which was obtained 72 hours prior to the initial CT examination using the isotope dilution mass spectrometry-traceable Modification in Diet in Renal Disease equation for GFR, which is based on the Kidney Disease Outcomes Quality Initiative recommendations of the National Kidney Foundation $[4,5]$. Before cancer therapy was initiated, the changes in renal function were assessed using both the sCr level and eGFR, which were collected after various protocol CT examinations (with and without imaging contrast agents) were performed. In patients for whom there were multiple sCr results in this 72-hour period, the $\mathrm{sCr}$ level that was measured closest to the date on which CT was performed was selected. The patients were stratified into subgroups according to their eGFR level: 90 or higher (Grade 5), 60-89 (Grade 4), 30-59 (Grade 3), 30-15 (Grade 2), and lower than $15 \mathrm{~mL} / \mathrm{min} / 1.73 \mathrm{~m}^{2}$ (Grade 1) to mirror the Kidney Disease Outcomes Quality Initiative guidelines for classifying CKD during the initial oncologic work-up examination before cancer therapy [4].

The sCr level-defined cut-off for AKI, which was created by the Acute Kidney Injury Network (AKIN) and is similar to the traditional definition of contrast-induced nephrotoxicity (CIN), was chosen for use in this study. The AKIN classification only relies on the sCr level and not on changes in the eGFR; the baseline sCr was not necessary and it required at least two $\mathrm{sCr}$ values that were obtained within a 48-hr period. AKI was defined as a sudden decrease (within $48 \mathrm{hr}$ ) in renal function, defined as an increase in the absolute $\mathrm{sCr}$ value of at least $26.5 \mu \mathrm{mol} / \mathrm{L}(0.3 \mathrm{mg} / \mathrm{dL})$ or as an increase in the $\mathrm{sCr}$ of $\geq 50 \%(1.5 \times$ the baseline value) or a decrease in the $\mathrm{UO}$ (documented oliguria $<0.5$ $\mathrm{mL} / \mathrm{kg} / \mathrm{h}$ for more than $6 \mathrm{hr}$ as an absolute $\mathrm{sCr}$ increase of $0.5 \mathrm{mg} / \mathrm{dLor}$ increase in the $\mathrm{sCr}$ value that was 1.25 -fold above the baseline value.

\section{Statistical analysis}

Continuous data with non-normal distributions are displayed as medians with inter-quartile ranges and were compared using the
Wilcoxon signed-rank test and comparative Student t-test. Categorical data are displayed as relative frequencies (percentages) and were compared using the two-by-two Fischer's exact test and Chi-square test. The risk factors of post-CT AKI and CKD were statistically

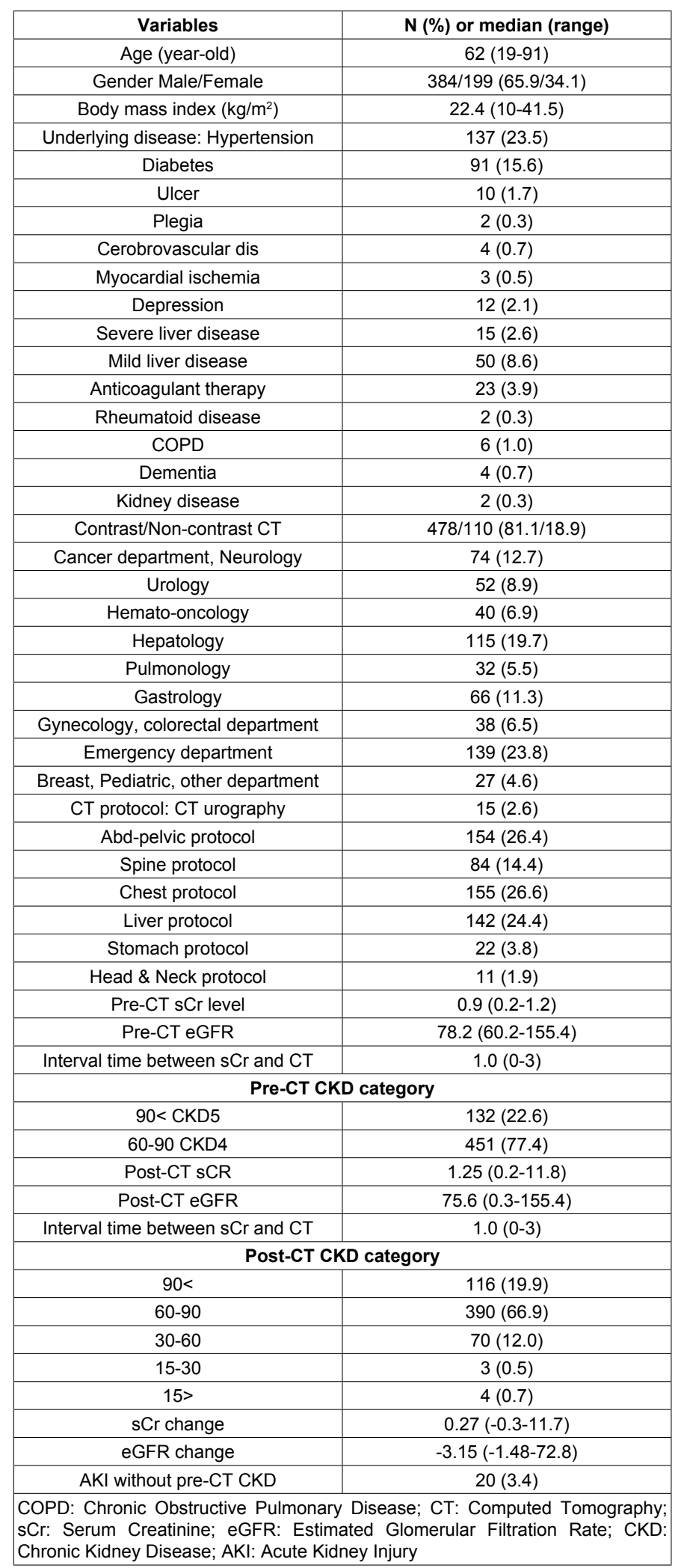

Table 1: Baseline demographics ( $\mathrm{N}=583)$. 
Citation: Kim SH, Joung JY, Seo HK, Lee KH, Chung J (2018) The Effect of the First Computed Tomography Examination on Renal Function During the Initial Cancer Work-up in Newly Diagnosed Cancer Patients without Chronic/Kidney Disease: A Retrospective, Single-institution, Observational Study. J Cancer Sci Ther 10: 332-337. doi: 10.4172/1948-5956.1000564

\begin{tabular}{|c|c|c|c|}
\hline Parameters & Non-AKI group & AKI group & p-value \\
\hline Age & $59.7 \pm 16.1$ & $57.6 \pm 17.5$ & 0.561 \\
\hline Gender, M/F & $368 / 195(65.4 / 34.6)$ & $16 / 4(80 / 20)$ & 0.232 \\
\hline BMI & $22.4 \pm 4.5$ & $21.9 \pm 6.3$ & 0.651 \\
\hline CCI & $4.9 \pm 2.2$ & $4.6 \pm 2.4$ & 0.520 \\
\hline Contrast CT & $459(81.5)$ & $14(70.0)$ & 0.239 \\
\hline Hypertension & $129(23.1)$ & $8(40.0)$ & 0.105 \\
\hline Diabetes & $87(15.6)$ & $4(20.0)$ & 0.537 \\
\hline Pre-CT sCr level & $0.9 \pm 0.2$ & $0.9 \pm 0.2$ & 0.836 \\
\hline Pre-CT eGFR & $82.9 \pm 23.8$ & $85.2 \pm 17.7$ & 0.031 \\
\hline Pre-CT interval time & $1.3 \pm 1.7$ & $2.1 \pm 1.8$ & 1.000 \\
\hline Pre-CKD 1/2 & $128 / 435(22.7 / 77.3)$ & $4 / 16(20 / 80)$ & 0.200 \\
\hline Post-CT sCr & $0.9 \pm 0.2$ & $8.6 \pm 25.9$ & 0.001 \\
\hline Post-CT eGFR & $80.1 \pm 22.4$ & $31.0 \pm 16.2$ & $1.8 \pm 1.2$ \\
\hline Interval time between sCr and CT & $1.5 \pm 1.0$ & 0.200 \\
\hline Post-CT CKD 1/2/3/4/5 & $116 / 390 / 57 / 0 / 0(20.6 / 69.3 / 10.1 / 0 / 0)$ & 7.2 & 0.001 \\
\hline sCr change & $0.96 \pm 0.1$ & $-54.2 \pm 24.0$ & 0.200 \\
\hline eGFR change & $-2.8 \pm 16.0$ & 0.001 \\
\hline
\end{tabular}

CT: Computed Tomography; AKI: Acute Kidney Injury; BMI: Body Mass Index; CCI: Charlson Comorbidity Index; sCr: Serum Creatinine; eGFR: Estimated Glomerular Filtration Rate; CKD: Chronic Kidney Disease

Table 2: Comparison of the baseline characteristics of patients with post-CT $A K I(N=20)$ and those without $A K I(N=564)$

\begin{tabular}{|c|c|c|c|}
\hline Parameters & CKD group & Non-CKD group & p-value \\
\hline Age & $62.8 \pm 14.7$ & $59.1 \pm 16.3$ & 0.060 \\
\hline Gender, M/F & $48 / 29(62.3 / 37.7)$ & $336 / 170(66.4 / 33.6)$ & 0.520 \\
\hline BMI & $23.1 \pm 4.3$ & $22.2 \pm 4.6$ & 0.143 \\
\hline $\mathrm{CCl}$ & $5.1 \pm 2.3$ & $4.8 \pm 2.2$ & 0.293 \\
\hline Contrast CT & $60(77.9)$ & $413(81.6)$ & 0.436 \\
\hline Underlying disease: Hypertension & $32(41.6)$ & $105(20.9)$ & 0.001 \\
\hline Diabetes & $19(24.7)$ & $72(14.3)$ & 0.028 \\
\hline Ulcer & $4(5.2)$ & $6(1.2)$ & 0.032 \\
\hline Anticoagulant therapy & $7(9.1)$ & $16(3.2)$ & 0.023 \\
\hline Pre-CT sCr level & $1.0 \pm 0.2$ & $0.9 \pm 0.2$ & 0.001 \\
\hline Pre-CT eGFR & $72.6 \pm 12.7$ & $84.6 \pm 24.5$ & 0.003 \\
\hline Pre-CT interval time & $1.8 \pm 1.9$ & $1.2 \pm 1.6$ & 0.10 \\
\hline Pre-CKD I/II & $4 / 73(5.2 / 94.8)$ & $128 / 378(25.3 / 74.7)$ & 0.001 \\
\hline Pre-CT CKD & -- & -- & -- \\
\hline Post-CT sCR & $3.1 \pm 13.4$ & $0.9 \pm 0.2$ & 0.149 \\
\hline Post-CT eGFR & $48.9 \pm 13.8$ & $82.9 \pm 21.9$ & 0.001 \\
\hline Interval time between sCr and CT & $1.5 \pm 1.0$ & $1.5 \pm 1.0$ & 0.553 \\
\hline Post-CT CKD 1, 2, 3, 4, 5 & $0 / 0 / 70 / 3 / 4(0 / 0 / 90.9 / 3.9 / 5.2)$ & $116 / 390 / 0 / 0 / 0(22.9 / 77.1 / 0 / 0 / 0)$ & 0.001 \\
\hline sCr change & $2.1 \pm 13.4$ & $0.0 \pm 0.1$ & 0.165 \\
\hline eGFR change & $-23.7 \pm 22.5$ & $-1.6 \pm 16.4$ & 0.001 \\
\hline AKI s pre-CT CKD & $20(26.0)$ & 0 & 0.001 \\
\hline
\end{tabular}

CT: Computed Tomography; CKD: Chronic Kidney Disease; BMI: Body Mass Index; CCI: sCr: Serum Creatinine; eGFR: Estimated Glomerular Filtration Rate; AKI: Acute Kidney Injury

Table 3: Comparison of baseline characteristics between patients with post-CT CKD $(N=77)$ and those without $C K D(N=507)$.

assessed using a logistic regression analysis, and a p-value less than 0.05 indicated statistical significance. All statistical analyses were performed using R (version 2.15; R Foundation for Statistical Computing, Vienna, Austria) [6] and STATA (release 9.2, STATA Inc., College Station, TX).

\section{Results}

The male-to female sex ratio of the 583 patients in this study was $454(62.1 \%) / 277(37.9 \%)$, and the mean overall body mass index and age were 23.7 (standard deviation \pm 27.7$) \mathrm{kg} / \mathrm{m}^{2}$ and $61.9( \pm 16.1)$ years, respectively. Initially, $136(18.6 \%)$ patients received contrast CT (CCT) or non-contrast CT (NCCT). In the CCT and NCCT groups, $20.5 \%$ and $19.1 \%$ of the patients, respectively, had CKD grade 3 . The mean sCr levels were significantly different between before and after CT was performed: $0.95( \pm 0.20) \mathrm{mg} / \mathrm{dLbefore} \mathrm{CT}$ and $1.23( \pm 4.5) \mathrm{mg} /$ dLafterwards $(\mathrm{p}<0.05)$, with a mean change in the $\mathrm{sCr}$ level of 0.27 $( \pm 4.47) \mathrm{mg} / \mathrm{dLand}$ eFGR change of $-3.15( \pm 18.00) \mathrm{mL} / \mathrm{min} / 1.73 \mathrm{~m}^{2}$ (Table 1). There was a mean of of $1.2 \pm 1.7$ days between the time CT was performed and the time at which the $\mathrm{sCr}$ level was measured. The percentage of patients who initially had post-CT AKI was $54.7 \%$ $(\mathrm{N}=400)$, including $36.8 \%$ of the NCCT group and $58.8 \%$ of the CCT group. Twenty (3.4\%) patients had an AKI grade of 4-5. The other baseline demographics are described in Tables 2 and 3.

The median sCrvalue $(0.96 \pm 0.20 v s .1 .06 \pm 0.45 \mathrm{mg} / \mathrm{dL})$ and eGFR (76.9 vs. $73.9 \mathrm{~mL} / \mathrm{min} / 1.73 \mathrm{~m}^{2}$ ) and their median changes between before and after CT (sCr, $0.27 \pm 4.47 \mathrm{mg} / \mathrm{dLand}$ eGFR $-3.15 \pm 18.00 \mathrm{~mL} /$ $\min / 1.73 \mathrm{~m}^{2}$ ) were not significantly different within a median interval 
Citation: Kim SH, Joung JY, Seo HK, Lee KH, Chung J (2018) The Effect of the First Computed Tomography Examination on Renal Function During the Initial Cancer Work-up in Newly Diagnosed Cancer Patients without Chronic/Kidney Disease: A Retrospective, Single-institution, Observational Study. J Cancer Sci Ther 10: 332-337. doi: 10.4172/1948-5956.1000564

\begin{tabular}{|c|c|c|c|c|}
\hline \multirow{2}{*}{ Variables } & \multirow{2}{*}{$p$-value } & \multirow{2}{*}{ Hazard ratio } & \multicolumn{2}{|c|}{$95 \%$ confidence interval } \\
\hline & & & Lower limit & Upper limit \\
\hline Non-Contrast CT & 0.038 & 0.619 & 0.394 & 0.973 \\
\hline Body mass index & 0.535 & 1.010 & 0.980 & 1.041 \\
\hline Male gender & $<0.001$ & 0.288 & 0.220 & 0.377 \\
\hline Age & 0.022 & 1.010 & 1.001 & 1.018 \\
\hline Hypertension & 0.014 & 1.464 & 1.080 & 1.984 \\
\hline Diabetes & 0.924 & 1.016 & 0.738 & 1.397 \\
\hline Ulcerative disease & 0.121 & 3.183 & 0.737 & 13.745 \\
\hline Warfarin therapy & 0.242 & 0.716 & 0.409 & 1.253 \\
\hline Pre-CT estimated GFR & $<0.001$ & 1.027 & 1.021 & 1.033 \\
\hline CT Protocol: CT urography & 0.001 & -- & -- & -- \\
\hline Abd-pelvic protocol & 0.759 & 0.832 & 0.256 & 2.704 \\
\hline Spine protocol & 0.249 & 0.581 & 0.231 & 1.462 \\
\hline Chest protocol & 0.838 & 0.901 & 0.332 & 2.447 \\
\hline Liver protocol & 0.768 & 0.871 & 0.350 & 2.172 \\
\hline Stomach protocol & 0.567 & 1.304 & 0.526 & 3.234 \\
\hline Head \& Neck protocol & 0.979 & 1.015 & 0.349 & 2.952 \\
\hline
\end{tabular}

Table 4: Risk factors of post-CT AKI in the multivariate analysis.

\begin{tabular}{|c|c|c|c|c|}
\hline \multirow{2}{*}{ Variables } & \multirow{2}{*}{$p$-value } & \multirow{2}{*}{ Hazard ratio } & \multicolumn{2}{|c|}{$95 \%$ confidence interval } \\
\hline & & & Lower limit & Upper limit \\
\hline CT protocol: CT urography & 0.024 & -- & -- & -- \\
\hline Abd-pelvic protocol & 0.288 & 0.276 & 0.026 & 2.959 \\
\hline Spine protocol & 0.059 & 0.123 & 0.014 & 1.080 \\
\hline Chest protocol & 0.253 & 0.256 & 0.025 & 2.644 \\
\hline Liver protocol & 0.505 & 0.491 & 0.060 & 3.984 \\
\hline Stomach protocol & 0.619 & 0.587 & 0.072 & 4.782 \\
\hline Head \& Neck protocol & 0.980 & 0.971 & 0.097 & 9.702 \\
\hline Contrast CT & 0.393 & 1.513 & 0.586 & 3.908 \\
\hline Pre-CT estimated GFR & 0.007 & 0.957 & 0.927 & 0.988 \\
\hline Body mass index & 0.462 & 1.026 & 0.958 & 1.098 \\
\hline Male gender & $<0.001$ & 0.082 & 0.038 & 0.175 \\
\hline Age & 0.049 & 1.022 & 1.000 & 1.044 \\
\hline Hypertension & 0.605 & 1.172 & 0.642 & 2.141 \\
\hline Diabetes & 0.330 & 1.395 & 0.713 & 2.729 \\
\hline Ulcerative disease & 0.859 & 0.894 & 0.260 & 3.070 \\
\hline Warfarin therapy & 0.036 & 0.371 & 0.147 & 0.937 \\
\hline
\end{tabular}

Table 5: Risk factors of post-CT CKD in the multivariate analysis.

time of 1.0 day between the CT and blood examinations ( $\mathrm{p}>0.05)$.

The rates of post-CT CKD grades 4-5 and AKI grades 4-5 were $13.2 \%(\mathrm{~N}=77)$ and $3.4 \%(\mathrm{~N}=20)$, respectively. The multivariate analysis showed that female sex (hazard ratio [HR] 0.757, 95\% confidence interval [CI] 0.589-0.974), the use of warfarin therapy (HR 0.371, 95\% CI 0.147-0.937), and pre-CT eGFR (HR 1.010, 95\% CI 1.004-1.017) were significant predictors of CKD. Female sex (HR 0.288, 95\% CI 0.220-0.377), age (HR 1.010, 95\% CI 1.001-1.018), hypertension (HR 1.464, 95\% CI 1.080-1.984), pre-CT eGFR (HR 1.027, 95\% CI 1.021 1.033), and no use of contrast media (HR 0.619, 95 CI 0.394-0.973) were significant predictors of AKI $(\mathrm{p}<0.05)$ (Tables 4 and 5$)$.

\section{Discussion}

This study investigated the nephrotoxic effect of the first outpatient-based diagnostic CT examination on the renal function of newly diagnosed cancer patients who waited for the initiation of cancer therapy after they were diagnosed with cancer during a health checkup screening program. Female sex, the use of warfarin therapy, and the pre-CT eGFR were significant predictors of CKD, and female sex, age, hypertension, pre-CT eGFR, and no use of a contrast agent were significant predictors of AKI.

Once cancer is diagnosed, diverse examining modalities, including blood tests and imaging, are performed. Until their cancer is properly treated, patients will undergo several non-friendly renal functional therapies and be exposed to $\mathrm{x}$-ray exposure several times to determine the disease stage and therapeutic follow-up protocol. The most popular and regularly performed work-up modality is CT, despite its negative effect on the patient's health because of radiating $\mathrm{x}$-rays and need for nephrotoxic contrast agents [7-9]. Deterioration in function is a frequently encountered health problem in cancer patients, and it may further affect the patient's therapeutic plan and quality of life after the cancer is completely cured.

There are two terms for deterioration in renal function during nephrotoxic therapy and work-up examinations. CKD is a term for deterioration in renal functional that results from continuous and repetitive renal impairment during cancer therapy $[4,10]$. In these 
Citation: Kim SH, Joung JY, Seo HK, Lee KH, Chung J (2018) The Effect of the First Computed Tomography Examination on Renal Function During the Initial Cancer Work-up in Newly Diagnosed Cancer Patients without Chronic/Kidney Disease: A Retrospective, Single-institution, Observational Study. J Cancer Sci Ther 10: 332-337. doi: 10.4172/1948-5956.1000564

patients, dialysis is initiated earlier and the maximal removal of uremic toxins is performed using a higher dosage and frequency of renal replacement therapy. There are also precautions for medications and food intake, even if the cancer is in remission. Another term is post-CT AKI, which is defined by comparing the post-CT sCr measurements to the pre-CT sCr values using the AKIN criteria [2,11]. The AKIN criteria standardize the definition of AKI for evaluating CIN (absolute $\mathrm{sCr}$ increase of $0.5 \mathrm{mg} / \mathrm{dLor}$ increase in the $\mathrm{sCr}$ by 1.25 -fold above the baseline value) $[2,8,11,12]$. The American College of Radiology recently advocated using the AKIN criteria to standardize the historically varied definitions of contrast-induced nephropathy in the literature [13].

CIN will occur in 2-25\% of patients undergoing coronary intervention and $0.5-5.5 \%$ of patients who undergo a CT examination $[7,10,14-16]$. The wide range of the incidence of CIN depends on the study design and definition that was used to identify those with a kidney injury. Contrast agents, which are a completely iatrogenic cause of renal failure, frequently cause hospital-acquired contrast-induced AKI (CI-AKI), [17] accounting for $11 \%$ to $12 \%$ of cases. Acute renal replacement will be required for $3 \%$ to $15 \%$ of CI-AKI patients [3]. Patients with CKD or AKI after undergoing CT are at a high risk of being hospitalized because of CI-AKI-related complications. They are predisposed to having continued loss of kidney function compared with patients with similar premorbid characteristics $[18,19]$. The high-risk patients who were analyzed in this study were hypertensive, older, and female, and had a higher baseline eGFR. In addition, these older, female patients with a poor baseline eGFR had a higher chance of developing CKD than did those who were younger, male, and had a better baseline eGFR. These at-risk patients would need to undergo non-contrast CT with protective measures during hospitalization before CT to prevent post-CT AKI and CKD. Anticoagulant therapy might help improve the patient's vasculature, with good patency for a hypervascular kidney, and help prevent thromboembolism, especially in those with underlying kidney and cardiovascular diseases $[7,10,14-16,20]$.

Previous studies have found that age, sex, the use of a contrast agent, hypertension, and anticoagulant therapy are significant risk factors of renal deterioration and CIN, $[14,18,19,21-23]$ similar to the results of this study. Other risk factors include diabetes mellitus, heart failure, anemia, hypotension, use of an intra-aortic balloon pump, left ventricular ejection fraction $<40 \%$, the type and dose of the contrast medium, a longer hospital stay, long-term dialysis, and in-hospital mortality [24,25].

Multiple studies showed that a difference in sex was a risk factor that significantly affected renal function. This is because testosterone and estrogen act reversely on renal excretion [26]. Especially for the excretion of nephrotoxic agents such as contrast agents, testosterone affects the expression of renal transport proteins, resulting in increased urinary excretion [26,27]. However, in this study, patients with AKI who underwent contrast-enhanced CT had good renal function without any predisposing renal diseases, and patients who received contrast agents were less likely to have AKI after CT than were those without contrast agents. However, the pre-CT eGFR had a negative effect upon AKI because those with a higher eGFR had a higher chance of undergoing contrast-enhanced CT than did those with a lower eGFR, greatly affecting the CT work-up examination.

The difficulty of planning a randomized controlled trial in which patients are randomized to received NCCT or CCT is difficult because it is unethical to randomize patients rather than to use clinical judgment to carefully balance the potential diagnostic benefits and risks of administering a contrast agent for specific clinical indications (not limited to AKI) and the risk factors of every individual patient [1]. However, recently published evidence strongly suggests that the risk that is caused by contrast material is much smaller than previously thought, based on non-controlled studies that were similar to ours. In patients with a baseline sCr value $<1.5 \mathrm{mg} / \mathrm{dLand}$ an eGFR $\geq 45 \mathrm{~mL} /$ $\min / 1.73 \mathrm{~m}^{2}$, the risk of CIN-AKI is likely to be nonexistent.

\section{Conclusion and Limitations}

This study had some limitations. First, large numbers of patients were excluded because of insufficient $\mathrm{sCr}$ data. Therefore, this outpatient population did not have follow-up sCr testing, and this study could have been favorably biased toward individuals with greater comorbidities and a propensity towards AKI. Second, the $\mathrm{sCr}$ level that was used for determining AKI was a less sensitive marker for renal function than eGFR; the peak sCr might occur in $15 \%$ of patients as long as 7 days after the administration of contrast-enhanced CT [28]. Third, there are no standardized protocols for the administration of either CCT or NCCT, but the treatment decision was dependent upon the clinician's discretion, which was based on either concern about AKI or because of the CT indication. However, this study was the first attempt to identify the nephrotoxic effect of the initial CT examination on renal function in newly treated cancer patients to define the overall nephrotoxic effect of imaging modalities and therapeutic drug toxicity on renal function during cancer therapy to elicit further information about the patient's quality of life after their cancer is in remission. A further longitudinal follow-up study should be performed to evaluate the final changes in renal function in cancer patients after they complete cancer therapy.

\section{Conflicts of Interest}

All the authors had no competing interests in this study.

\section{Financial Disclosure}

This study was supported by the Korean National Cancer Center Grants (nos. 1810242-1)

\section{References}

1. Meinel FG, DeCecco CN, Schoepf UJ, Katzberg R (2014) Contrast-induced acute kidney injury: Definition, epidemiology, and outcome. BioMed Res Int 2014: 859328

2. Lopes JA, Jorge S (2013) The RIFLE and AKIN classifications for acute kidney injury: a critical and comprehensive review. Clin Kidney J 6: 8-14.

3. Sinert R, Brandler E, Subramanian RA, Miller AC (2012) Does the current definition of contrast-induced acute kidney injury reflect a true clinical entity? Acad Emerg Med 19: 1261-1267.

4. Levey AS, Coresh J, Bolton K, Culleton B, Harvey KS, et al. (2002) K/DOQ clinical practice guidelines for chronic kidney disease: evaluation, classification, and stratification. Am J Kid Dis 39: S1-S266.

5. Levey AS, Bosch JP, Lewis JB, Greene T, Rogers N, et al. (1999) A more accurate method to estimate glomerular filtration rate from serum creatinine: a new prediction equation. Modification of diet in renal disease study group. Ann Intern Med 130: 461-470.

6. R-Development Core Team (2012) R: A language and environment for statistical computing.

7. Barrett BJ, Katzberg RW, Thomson HS, Chen N, Sahani D, et al. (2006) Contrast-induced nephropathy in patients with chronic kidney disease undergoing computed tomography: A double-blind comparison of iodixanol and iopamidol. Invest Radiol 41: 815-821.

8. Davenport MS, Khalatbari S, Cohan RH, Dillman JR, Myles JD, et al. (2013) Contrast material-induced nephrotoxicity and intravenous low-osmolality iodinated contrast material: Risk stratification by using estimated glomerular filtration rate. Radiology 268: 719-728. 
Citation: Kim SH, Joung JY, Seo HK, Lee KH, Chung J (2018) The Effect of the First Computed Tomography Examination on Renal Function During the Initial Cancer Work-up in Newly Diagnosed Cancer Patients without Chronic/Kidney Disease: A Retrospective, Single-institution, Observational Study. J Cancer Sci Ther 10: 332-337. doi: 10.4172/1948-5956.1000564

9. Heidenreich A, Desgrandschamps F, Terrier F (2002) Modern approach of diagnosis and management of acute flank pain: review of all imaging modalities. Eur Eurol 41: 351-362.

10. Anonymous (2010) Notice of redundant publication. Am J Kidney Dis 55: 639-647.

11. Lakhal K, Ehrmann S, Chaari A, Laissy JP, Regner B, et al. (2011) Acute kidney injury network definition of contrast-induced nephropathy in the critically ill: incidence and outcome. J Crit Care 26: 593-599.

12. Valette X, Parienti JJ, Plaud B, Lehoux P, Samba D, et al. (2012) Incidence, morbidity, and mortality of contrast-induced acute kidney injury in a surgical intensive care unit: a prospective cohort study. J Crit Care 27: e1-5.

13. Committee ACoR (2012) American college of radiology committee on drugs and contrast media: Reston, Va.

14. Mehran R, Aymong ED, Nikolsky E, Lasic Z, lakovou I, et al. (2004) A simple risk score for prediction of contrast-induced nephropathy after percutaneous coronary intervention: development and initial validation. J Am Coll Cardiol 44: 1393-1399.

15. Lencioni R, Fattori R, Morena G, Stacul F (2010) Contrast-induced nephropathy in patients undergoing computed tomography (CONNECT) - A clinical problem in daily practice? A multicenter observational study. Acta Radiol 51: 741-750.

16. Weisbord SD, Mor MK, Resnick AL, Martwig KC, Palevsky PM, et al. (2008) Incidence and outcomes of contrast-induced AKI following computed tomography. Clin J Am Soc Nephrol 3: 1274-1281.

17. Shusterman N, Strom BL, Murray TG, Morrison G, West SL, et al. (1987) Risk factors and outcome of hospital-acquired acute renal failure. Clinical epidemiologic study. Am J Med 83: 65-71.

18. McCullough PA, Wolyn R, Rocher LL, Levin RN, O'Neill WW (1997) Acute renal failure after coronary intervention: incidence, risk factors, and relationship to mortality. Am J Med 103: 368-375.
19. Parfrey PS, Griffiths SM, Barrett BJ, Paul MD, Genge M, et al. (1989) Contrast material-induced renal failure in patients with diabetes mellitus, renal insufficiency, or both. A prospective controlled study. N Engl J Med 320: 143-149.

20. Gigante A, Barbano B, Sardo L, Martina P, Gasperini ML, et al. (2014) Hypercoagulability and nephrotic syndrome. Curr Vasc Pharmacol 12: 512-517.

21. Rihal CS, Textor SC, Grill DE, Berger PB, Ting HH, et al. (2002) Incidence and prognostic importance of acute renal failure after percutaneous coronary intervention. Circulation 105: 2259-2264.

22. Schwab SJ, Hlatky MA, Pieper KS, Davidson CJ, Morris KG, et al. (1989) Contrast nephrotoxicity: a randomized controlled trial of a nonionic and an ionic radiographic contrast agent. $\mathrm{N}$ Engl J Med 320: 149-153.

23. McDonald JS, McDonald RJ, Carter RE, Katzberg RW, Kallmes DF, et al (2014) Risk of intravenous contrast material-mediated acute kidney injury: a propensity score-matched study stratified by baseline-estimated glomerular filtration rate. Radiology 271: 65-73.

24. Hong SI, Ahn S, Lee YS, Kim WY, Lim KS, et al. (2016) Contrast-induced nephropathy in patients with active cancer undergoing contrast-enhanced computed tomography. Support Care Cancer 24: 1011-1117.

25. Huang MK, Hsu TF, Chiu YH, Chiang SC, Kao WF, et al. (2013) Risk factors for acute kidney injury in the elderly undergoing contrast-enhanced computed tomography in the emergency department. J Chin Med Assoc 76: 271-276.

26. Johansen $\mathrm{KL}$ (2004) Testosterone metabolism and replacement therapy in patients with end-stage renal disease. Semin Dial 17: 202-208.

27. He R, Ai L, Zhang D, Wan L, Zheng T, et al. (2016) Different effect of testosterone and oestrogen on urinary excretion of metformin via regulating OCTs and MATEs expression in the kidney of mice. J Cell Mol Med 20: 2309-2317.

28. Mitchell AM, Jones AE, Tumlin JA, Kline JA (2010) Incidence of contrastinduced nephropathy after contrast-enhanced computed tomography in the outpatient setting. Clin J Am Soc Nephrol 5: 4-9. 